\title{
MYASTHENIC CRISIS- ANALYSIS OF PREDISPOSING FACTORS, CLINICAL FEATURES, COMPLICATIONS AND TREATMENT OUTCOME
}

\author{
Jayakumar Madavan1, Thamilpavai Natarajan², Lakshmi Narasimhan Ranganathan³, Bhanu Kesavamoorthy4
}

${ }^{1}$ Senior Assistant Professor, Department of Neurology, Institute of Neurology, Madras Medical College and Rajiv Gandhi Government General Hospital, Chennai, Tamilnadu, India.

2Senior Assistant Professor, Department of Neurology, Institute of Neurology, Madras Medical College and Rajiv Gandhi Government General Hospital, Chennai, Tamilnadu, India.

${ }^{3}$ Director and HOD, Department of Neurology, Institute of Neurology, Madras Medical College and Rajiv Gandhi Government General Hospital, Chennai, Tamilnadu, India.

${ }^{4}$ Former Director and HOD, Department of Neurology, Institute of Neurology, Madras Medical College and Rajiv Gandhi Government General Hospital, Chennai, Tamilnadu, India.

ABSTRACT
BACKGROUND
Myasthenia gravis is a neuromuscular junction disorder characterised by skeletal muscle weakness and fatigability. If the
respiratory muscles and/ or bulbar muscles become weak, it requires respiratory assistance and the patient is said to be in
myasthenic crisis.

\section{MATERIALS AND METHODS}

In this descriptive study, patients admitted between 01.10.2012 and 01.12.2013 in Rajiv Gandhi Government General Hospital, Madras Medical College with symptoms and signs of myasthenia crisis, fulfilling the inclusion and exclusion criteria were enrolled with informed consent.

\section{RESULTS}

Breathing difficulty was the predominant symptom in all the patients (96\%) followed by speech (88\%) and chewing (84\%). Our patients had a minimum disease duration of 7 days and maximum of 1460 days and a mean of 627.16 days. 6 patients had thymoma. The most common predisposing factor was respiratory infection (28\%). The duration of IMCU stay also ranged from 3 days to 40 days with a mean of 13.32 days. Three patients developed ventilator-associated pneumonia (12\%). One patient developed respiratory failure, autonomic dysfunction and shock within 5 days of crisis.

\section{CONCLUSION}

The better survival rate in ventilated patients outweighs the preventable ventilator related complications. Standardised myasthenia crisis treatment protocol and advanced neurointensive care setup minimises the negative impact of the co-morbid conditions on myasthenic crisis. Therapeutic regimens should be tailored to the patient's age, severity of crisis, circulatory status and comorbid conditions. Fixed standardised and combined treatment regimen is not suitable for each and every patient.

\section{KEY WORDS}

Myasthenic Crisis, Thymoma.

HOW TO CITE THIS ARTICLE: Madavan J, Natarajan T, Ranganathan LN, et al. Myasthenic crisis-analysis of predisposing factors, clinical features, complications and treatment outcome. J. Evolution Med. Dent. Sci. 2018;7(22):2681-2685, D0I: $10.14260 /$ jemds/2018/604

\section{BACKGROUND}

Myasthenia gravis is a neuromuscular junction disorder characterised by skeletal muscle weakness and fatigability. It is due to a decrease in the number of available acetylcholine receptors at the neuromuscular junction as a result of antibody-mediated autoimmune attack. In myasthenia gravis, the defect is a decrease in the number of available AchRs at

'Financial or Other Competing Interest': None.

Submission 31-01-2018, Peer Review 13-05-2018,

Acceptance 21-05-2018, Published 28-05-2018.

Corresponding Author:

Thamilpavai Natarajan,

Senior Assistant Professor,

Department of Neurology,

Madras Institute of Neurology,

Medical College and Rajiv Gandhi Government

General Hospital, Park Town, Chennai-3,

Tamilnadu.

E-mail: arulpavai@gmail.com

DOI: $10.14260 /$ jemds $/ 2018 / 604$ the postsynaptic muscle membrane and the postsynaptic folds are flattened resulting in decreased efficiency of neuromuscular transmission. Even though Ach is released normally, it produces small end plate potentials that fails to trigger muscle action potentials. Failure of transmission at multiple neuromuscular junctions causes weakness of muscle contraction. Many subtypes of myasthenia gravis are identified basing on clinical presentation, age of onset, thymic pathology and autoantibody profile. ${ }^{[1]}$ Myasthenic crisis (MC) is an uncommon life-threatening neurological emergency. [2-4]

It may occur in known myasthenia gravis patients, generally during the first year after diagnosis or as the presentation itself. The hallmark of myasthenic crisis is the bulbar and/ or respiratory weakness. Management of these patients poses a great challenge due to its fluctuating nature and clinical course. Prevention and treatment of myasthenic crisis requires admission to a neuroscience ICU, vigilant care and when necessary intubation for ventilatory and feeding support. Acute management lies not only in ventilator care, but also by reducing circulating antibody titres with 
immunological therapy such as plasmapheresis (PE), immunoglobulin (IVIg) and corticosteroids followed subsequently by oral steroids and immunotherapy in the long run with frequent monitoring of the complications associated with these drug therapies. Repeated courses of plasmapheresis (PE) and immunoglobulin (IVIg) can be given in patients with refractory myasthenic crisis even after control of predisposing factors.

\section{Aim of the Study}

To study the predisposing factors, clinical features, associated complications and treatment outcome in patients with myasthenic crisis.

\section{MATERIALS AND METHODS}

In this descriptive study, patients admitted between 01.10.2012 and 01.12.2013 in the Neurology ward, Medical ward and IMCU of Rajiv Gandhi Government General Hospital, Madras Medical College with symptoms and signs of myasthenic crisis, fulfilling the inclusion and exclusion criteria were enrolled after explaining and getting consent for the study. Institutional Ethical Committee approval was obtained.

\section{Inclusion Criteria}

Patients with symptoms and signs of myasthenic crisis and admitted in Neurology ward, Medical ward and IMCU are registered for the study.

\section{Exclusion Criteria}

1. Patients who are diagnosed to have other medical and surgical conditions.

2. Other co-morbid conditions like HIV, malignancy.

3. Congenital myasthenic syndrome.

4. Renal failure.

5. Hepatic failure.

6. Patients with preoperative respiratory crisis associated with thymectomy.

7. Pregnant patients.

\section{Methods and Analysis}

Patients in myasthenic crisis who were admitted in Neurology ward, Medical ward and IMCU were enrolled. Detailed history and physical examination findings were recorded. All baseline investigations were done.

X-ray Chest, CT-Chest, MRI imaging of Brain and Spinal cord were done. ENMG and repetitive nerve stimulation were done in all patients. Anti-Acetylcholinesterase antibodies and thyroid function tests were done in all patients and anti-musk antibodies when indicated.

\section{The following parameters were analysed-}

- Demographic data.

- Duration of illness.

- Predisposing factors.

- Clinical features.

- Associated complications.

- Duration of mechanical ventilation.

- Ventilator associated complications.

- Treatment received.

- Outcome of patients.

\section{Statistical Analysis}

All data were analysed using SPSS version 20.0. Chi-square test is used for goodness of fit and independence of attributes.

\section{RESULTS}

In our study 4 patients were below 30 years of age, 9 in 3140 years' age group, about 10 of them in $41-60$ years and only 2 of them were above 60 years.

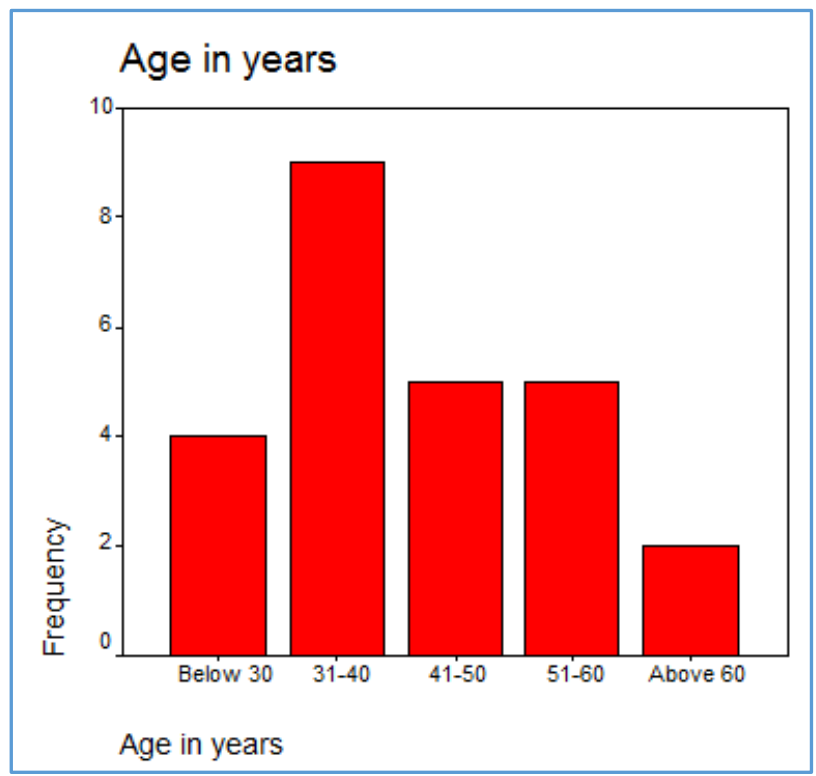

Chart-1

\section{Sex Distribution}

Among 25 patients, 14 were males and 11 were females.

\section{Predisposing Factor}

Out of 25 patients treated, predisposing factors were elicitable in only 15 of them. 2 of them had aspiration. 3 of them had viral fever, which caused worsening of their disease course. 1 patient had enteric fever. Upper respiratory tract infection in 6 of them. 1 patient presented with urosepsis. 1 myasthenic paient had coexistent spinal tuberculosis and was under category 1 ATT. 1 patient had lobar pneumonia demonstrated by chest x-ray and HRCT chest. During that period patient had worsening of myasthenia gravis and susequently developed crisis.

\section{Clinical Profile}

All of them presented with bulbar symptoms in addition to ocular weakness and generalised limb weakness. Nasal regurgitation was found in 12 patients in addition to ocular and generalised limb weakness, dysphagia in 19, 21 had chewing difficulty. All of them had breathing difficulty. 10 patients had a nasal twang during their speech signifying significant bulbar dysfunction. All of them had fatigable limb weakness with a limb power MRC grading of $3 / 5$ to $4 / 5$. Bilateral ptosis was found in 19 of them and 14 patients had diplopia.

\section{Disease Duration and Myasthenic Crisis}

The interval from symptom onset to crisis ranged from 3 - 7 days in 7 of them, 8 - 14 days in another 7. The duration of intensive medical care stay ranged from 3 days to one month. 
Once the patiient stabilised, they were shifted to general ward. Some of them were on non-invasive ventilation at admission and on desaturation connected to mechanical ventilation and few patients required ventilatory assistance on admission itself.

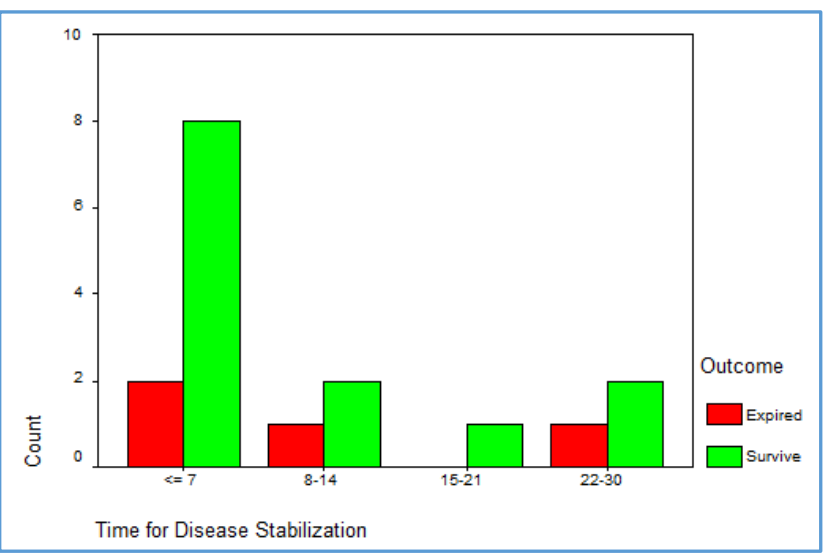

Chart 2. Time for Disease Stabilisation

\section{Thymoma and Crisis}

Thymoma screening was done in all of them by CT chest imaging. 19 of them had no thymoma, 6 of them had thymoma. Thymectomy was advised in all 25 patients after clinical stabilisation. Only 3 of them underwent thymectomy in thymomatous group and 5 of them in non-thymomatous group. Thymectomy was planned at a later date in the remaining 17 patients.

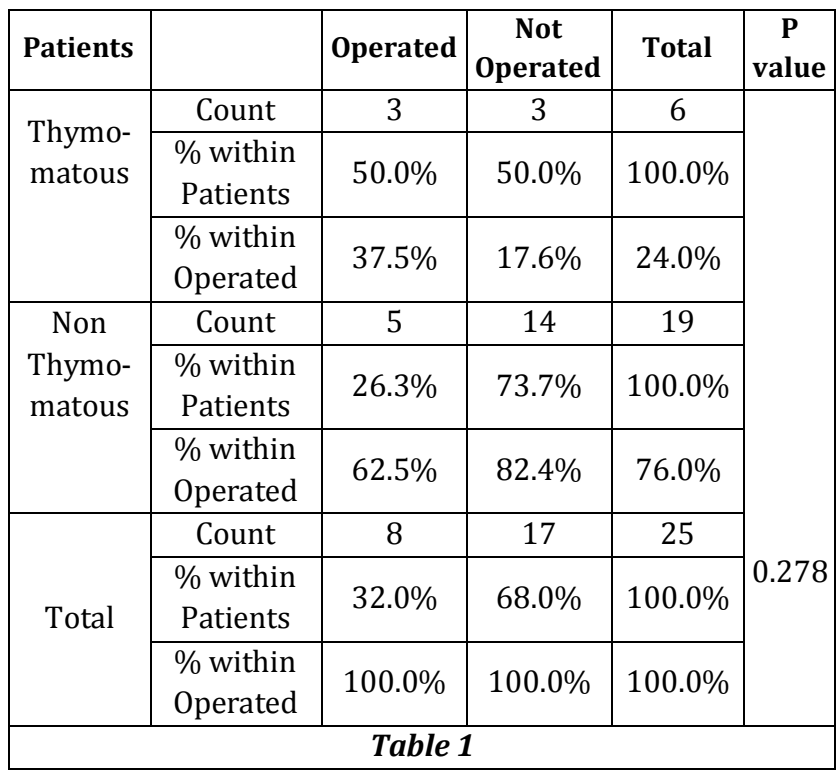

\section{Treatment}

All 25 patients were stopped of their oral prednisolone and started on IV methylprednisolone in a dose of $1 \mathrm{~g}$ IV for 5 days. IVIG was given in 3 patients at a dose of $0.4 \mathrm{mg} / \mathrm{kg}$ body weight after cardiac and renal assessment and in the absence of contraindications for a total of 5 days continuously after plasmapheresis. So 3 patients underwent both plasmapheresis and IVIG. 15 patients underwent only plasmapheresis 2 - $3 \mathrm{~L} /$ day on alternate days after cardiac and renal assessment and after their sepsis was under control. 7 patients improved with IV steroids and did not require either plasmapheresis or IVIG.

\begin{tabular}{|c|c|c|}
\hline & Frequency & Percent \\
\hline Both IVIG and plasmapheresis & 3 & 12.0 \\
\hline No IVIG or plasmapheresis & 7 & 28.0 \\
\hline Plasmapheresis done & 15 & 60.0 \\
\hline Total & $\mathbf{2 5}$ & $\mathbf{1 0 0 . 0}$ \\
\hline \multicolumn{2}{|c|}{ Table 2 } \\
\hline
\end{tabular}

\section{Complications and Treatment Outcome}

3 patients who were treated with both plasmapheresis and IVIG and 2 of them completely recovered. The other patient showed initial improvement, but later deteriorated and died a month later. 15 patients underwent plasmapheresis, 14 of them completely recovered. 1 among them died after completion of three cycles. 4 out of 25 patients died. Among these patients, 5 of them were diabetics and one patient had hypertension.

\section{DISCUSSION}

Myasthenic crisis is very often encountered in IMCU as a neurological emergency. Myasthenic crisis can involve the upper airway muscles, respiratory muscles inspiratory and expiratory, manifesting as dyspnoea. Signs of bulbar weakness include dysphagia, nasal regurgitation, slurred speech, jaw weakness, bifacial paresis, neck muscle weakness and tongue weakness. Respiratory support is the mainstay of management of patients in myasthenic crisis. Non-invasive ventilation (NIV) may be used to prevent or delay mechanical ventilation as extubation and weaning becomes very difficult in patients in myasthenic crisis. Treatment of myasthenic crisis is very cost effective as it requires ventilator support, IVIG, plasmapheresis, technical expertise and skilled nursing care.

In our study, the minimum age of onset of crisis is 17 years and maximum age of onset is 65 years. The maximum number of cases ( 9 patients) occurred in the age group of 3040 with male predominance ( 5 males and 4 females). Below 30 years 4 patients. 5 patients in 40 - 50 years' age group [3 females and 2 males], 5 in 50 - 60 years [ 4 females and 1 male] and 2 female patients above 60 years.

Sex distribution of crisis was $56 \%$ for males (14 cases) and $44 \%$ for females (11 cases). As per epidemiological data, women are twice as likely as men to be affected. MG shows a bimodal distribution with male: female ratio of 3: 7 if aged $<40$ years, 1: 1 if aged $40-49$ years, 3: 2 if aged $>50$ years by Godoy DA et al.[4] But in our study, there is a clear male preponderance.

\section{Predisposing Factors}

The most common predisposing factor in our patients were respiratory infection (28\%), which includes both upper and lower respiratory tract infection. The most common precipitant is infection as by Linda C. Wendell.[3] One series documented infection in $38 \%$ of patients presenting with myasthenic crisis; most commonly, the infection was bacterial pneumonia followed by a bacterial or viral upper respiratory infection. The other predisposing factors were aspiration pneumonitis, tuberculosis, urinary tract infection, enteric fever and viral fever. Certain drugs like quinolones, macrolide antibiotics can cause worsening of myasthenia gravis, but in our study there was no significant drugs attributable to the worsening. Predisposing factor is the most important and independent risk factor against the duration and severity of pre-existing myasthenia gravis. 


\section{Clinical Profile}

The severity of myasthenia gravis was assessed by Quantitative myasthenia gravis score and Osserman and Genkins classification and graded accordingly for prompt care and management. Breathing difficulty was the predominant symptom in all the patients $(96 \%)$ followed by speech difficulty (88\%) and chewing difficulty (84\%). Limb and generalised weakness is also another predominant symptom $(80 \%)$ in all the patients. Other symptoms like ptosis (76\%), dysphagia (76\%), diplopia (56\%), nasal regurgitation (48\%) and facial weakness $(28 \%)$ are in the decreasing frequency. Our patients presented with predominant bulbar symptoms similar to other studies. Some patients presented at onset itself with symptoms of crisis and later diagnosed and confirmed to have myasthenia gravis by clinical assessment, electrophysiology and antibody titres. Patients had an asymmetrical ptosis with preserved pupillary responses with extraocular muscle weakness. Bulbar dysfunction in these patients alters the cough reflex swallowing mechanisms and they had difficulty in handling oropharyngeal secretions, leading to alterations and increase the likelihood of microaspiration and pneumonitis. All patients underwent electrophysiological testing and also phrenic nerve studies to assess diaphragmatic involvement. Serological testing of Anti-AChR antibodies was also done for all patients.

\section{Disease Duration and Crisis}

Our patients had minimum disease duration of 7 days and maximum of 1460 days and a mean of 627.16 days. The interval from symptom to the onset of crisis was 7 days (minimum) and maximum of 1460 days with a mean of 439.36 days. The duration of crisis ranged from 2 to 30 days with a mean of 9.64 days. The duration of myasthenia gravis does not influence the occurrence of myasthenic crisis as reflected by the Pearson poor correlation with an r-value of .043. Our study correlates with other studies. Myasthenic crisis was the presenting symptom in three patients.

\section{Time for Disease Stabilisation and IMCU Stay}

Patients were on mechanical ventilation for a minimum of 2 days and a maximum of 30 days. 2 patients died within one week and another on the $30^{\text {th }}$ day of mechanical ventilation and another within a week after admission. 8 patients stabilised with non-invasive ventilation. The duration of IMCU stay also ranged from 3 days to 40 days with a mean of 13.32 days.

\section{Thymoma and Crisis}

In non-thymomatous MG, thymectomy is performed as an elective procedure to potentially minimise the dose of drugs or duration of immunotherapy or failure to respond to an initial trial of immunotherapy or have deleterious side-effects from drug therapy and in an effort to achieve medication-free remission in MG. Because of the long delay in onset of effect, thymectomy for MG is an elective procedure. It should be performed once the patient is stable and deemed safe to undergo a procedure where postoperative pain and mechanical factors can limit respiratory function. 6 patients had thymoma. Among them 3 were operated and 3 patients were advised to undergo operation. One patient who had thymoma was on prolonged ventilation for 30 days with ventilator associated pneumonia (VAP) died on the $30^{\text {th }}$ day. Thymectomy was done in 5 patients with non-thymomatous MG. Though thymectomy was carried out on these patients, the beneficial effect of thymectomy will take longer than 1 to 2 years.

\section{Treatment and Complications}

All our study patients were on oral steroids and on immunosuppressive agents. With the onset of crisis, they were stopped of oral steroids and switched over to IV Methylprednisolone and then on IVIG or plasmapheresis. As per the International consensus guidance for management of myasthenia gravis by Sanders DB neurology 2016 Jul 26; 87 (4): 419-425, as well by the Cochrane database 11, 12, PLEX and IVIg are appropriately used as short-term treatments in patients with MG with life-threatening signs such as respiratory insufficiency or dysphagia; in patients with significant bulbar dysfunction; when a rapid response to treatment is needed; when other treatments are insufficiently effective; and prior to beginning corticosteroids if deemed necessary to prevent or minimise exacerbations. The choice between PLEX and IVIg depends on individual patient factors (e.g. PLEX cannot be used in patients with sepsis and IVIg cannot be used in renal failure) and on the availability of each. IVIg and PLEX are probably equally effective in the treatment of severe generalised MG.[5]

In accordance with the guidelines, 15 of the 25 patients underwent plasmapheresis and 3 of them were given both plasmapheresis and IVIG. IVIG was given in a dose of 0.4 $\mathrm{mg} / \mathrm{kg}$ bodyweight for a period of 5 days under stringent monitoring. Plasmapheresis was done for 5 cycles on alternate days. Complications which occurred during the procedure in some patients were managed appropriately.

\section{Complications}

Three patients developed ventilator associated pneumonia (12\%). Among them one patient developed respiratory failure, autonomic dysfunction and shock within 5 days of crisis. Another patient developed respiratory failure, pneumonia with pleural effusion and collapse of underlying lobe and pneumothorax on the opposite side with subcutaneous emphysema within 10 days of crisis. All those patients who developed VAP were on prolonged ventilation (30 days). Comprehensive standard pulmonary and ventilatory care and hospital antibiotic usage guidelines can prevent these complications.

\section{Outcome}

The outcome was good recovery. 4 of them died due to ventilator associated complications. Advancement in the neurocritical care management techniques and the availability of immunomodulation therapy have drastically reduced the mortality rates of myasthenic crisis. A recent study suggests that aggressive respiratory treatment (use of suction, intermittent positive pressure breathing or bronchodilator treatments, sighs and chest physiotherapy) can lower the risk of atelectasis and ventilator-associated pneumonia. Before 1955, myasthenic crisis was fatal in $80 \%$ of episodes JMK Murthy and AK Meena.[6] In the recent Columbia series the mortality rate was $10 \%$ overall, half of those dying after extubation.[4] In the Columbia series, all deaths were attributable to severe medical comorbidity. The 
mortality in our series was $12 \%$ due to infection due to ventilator associated complications. This is comparable to western data where the overall in-hospital mortality rate was $2.2 \%$, being higher in MG crisis (4.47\%). Older age and respiratory failure were the predictors of death with adjusted odds ratios of 9.28 (95\% confidence interval [CI], 3.31, 26.0) and 3.58 (95\% CI, 2.01, 6.38).[7] Higher age is a risk factor and lesser age is a favourable factor for good outcome. Duration of myasthenia gravis does not have any role on the outcome of the patient. IMCU stay is a variable factor for the outcome of myasthenic patients.

Though patients on prolonged ventilation may be prone for VAP and other ventilator related complications, the better survival rate in ventilated patients outweigh the preventable ventilator related complications. Commonly in olden days, comorbid conditions had a major negative impact on the outcome of the myasthenic crisis.

\section{CONCLUSION}

1. In our study, the commonest age group of presentation of myasthenic crisis was in the fourth decade (36\%) with a male predominance (56\%).

2. The most common predisposing factor was respiratory infection (28\%).

3. In three patients, the presenting symptoms of myasthenia gravis were of crisis itself.

4. The mean duration of onset of myasthenia gravis to the onset of crisis was 439.36 days.

5. The duration of myasthenia gravis does not influence the occurrence of myasthenic crisis.

6. Our patients presented with predominant bulbar symptoms with respiratory distress.

7. Ventilator associated pneumonia and other respiratory complications influenced the outcome of treatment of myasthenic crisis.

8. $32 \%$ of thymectomised patients had myasthenic crisis.

9. Higher age is a risk factor and younger age is a favourable factor for good outcome.
10. The better survival rate in ventilated patients outweighs the preventable ventilator related complications.

\section{Limitation of the Study}

The treatment decision between PE and IVIg was not randomised and was based on the availability to the patients. Despite such limitations, both PE and IVIg are equally effective and safe. Better outcomes are achieved by earlier identification, decreasing the duration of intubation and also aggressively treating the associated medical comorbidity and complications. Patient's education is a must and are asked to report earlier if bulbar or respiratory distrust occurs.

\section{REFERENCES}

[1] Gajdos P, Chevret S, Toyka K. Intravenous immunoglobulin for myasthenia gravis. Cochrane Database Syst Rev 2008;(1):CD002277.

[2] Jani-Acsadi A, Lisak RP. Myasthenic crisis: guidelines for prevention and treatment. Journal of the Neurological Sciences 2007;261(1-2):127-33.

[3] Wendell LC, Levine JM. Myasthenic crisis. The Neurohospitalist 2011;1(1):16-22.

[4] Godoy DA, Mello LJ, Masotti L, et al. The myasthenic patient in crisis: an update of the management in Neurointensive Care Unit. Arquivos de NeuroPsiquiatria 2013;71(9A):627-39.

[5] Sanders DB, Wolfe GI, Benatar M, et al. International consensus guidance for management of myasthenia gravis: executive summary. Neurology 2016;87(4):419-25.

[6] Murthy JM, Meena AK, Chowdary GV, et al. Myasthenic crisis: clinical features, complications and mortality. Neurology India 2005;53(1):37-40.

[7] Alshekhlee AM, Miles JD, Katirji B, et al. Incidence and mortality rates of myasthenia gravis and myasthenic crisis in US hospitals. Neurology 2009;72(18):154854. 